\title{
Peutz-Jeghers syndrome presenting with non-small cell lung cancer
}

\begin{abstract}
Peutz-Jeghers syndrome (PJS) formerly known as Hereditary Intestinal Polyposis Syndrome is an autosomal dominant disorder associated with a mutation in the STK11 gene localized at 19p13.3. ${ }^{1} \mathrm{PJS}$ is most commonly associated gastrointestinal harmartomatous polyps and mucocutaneous pigments macules. ${ }^{2}$ Patients with PJS are at an increased risk for breast, intestinal, cervical, pancreatic, and testicular cancer. ${ }^{2}$ In a Dutch cohort study of PJS patients the increased risk of cancer was found to be 8.96 (CI 6.46 to 12.42) and greater in females compared to males with HR of 20.40 and 4.76 respectively (95\% CI 13.43 to 30.99 for female patients and 2.82 to 8.04 for male). ${ }^{3}$ Patients also tend to present earlier with cancer (average age 44 ) and have an increased risk of mortality. ${ }^{3}$

In this case we present a case of cancer in previously undiagnosed PJS. A middle-aged woman presented with cough, pleuritic, chest pain, and weight loss over three months. She had a positive family history for Peutz-Jeghers Syndrome. She was found to have squamous cell cancer of the lung. Recent research has illustrated a connection between one of the Peutz-Jeghers mutations, LKB1 (STK11:locus 19p13.3) and lung cancers in Caucasian populations.

While colorectal, breast, pancreatic, stomach, and ovarian cancer are the types of cancer most commonly associated with PJS, recent research suggests a genetic association with bronchioalveolar cancer. The STK11; locus 19p13.3 is also known as LKB1 mutation. Nearly half of all non-small cell lung cancer cell lines and one third of lung adenocarcinomas in Caucasian populations have the LKB1 mutation. ${ }^{46}$ This case represents a presentation of one such patient.
\end{abstract}

Volume 8 Issue 2 - 2018

\author{
Moneeb Sefeldawia Mustafa', Kara Kimball², \\ Khalid Yousif', Mohamed Zain' \\ 'St. Luke's Hospital Kilkenny, Ireland \\ ${ }^{2}$ University of Limerick Graduate Entry Medical School, Ireland
}

Correspondence: Moneeb Sefeldawia Mustafa, St. Luke's Hospital Kilkenny, Ireland,

Email muneeebseif@hotmail.com

Received: February 27, 2018 | Published: March 07, 2018

\section{Learning points}

a. Physicians should have a low-threshold for cancer screening in patients with PJS and family history of PJS.

b. The STK11; locus 19p13.3 mutation in Peutz-Jeghers Syndrome is also present in lung cancers in Caucasian populations. Lung cancer should be considered in patients presenting with non-specific symptoms who have a diagnosis of PJS or a family history of PJS

\section{Case report}

A middle aged woman presented to the medical assessment unit complaining of a 3-month history of cough and pleuritic chest pain. She reported wheeze and occasional scant sputum but denied hemoptysis. The chest pain was associated with a low grade fever and weight loss of approximately 5 kilograms in the same time period. She was seen in the community by her General Practitioner and treated with Augmentin and steroids to no relief.

Her medical history is non contributory, however, she had cholecystitis and multiple gallstones. She was not any medications. Her family history was relevant for Peutz-Jeghers Syndrome (PJS) in her mother who passed away from pancreatic cancer and two children with the syndrome.

\section{Examination and investigation findings}

Her physical exam revealed no abnormalities with a regular rate and rhythm of the cardiovascular system and clean and equal air entry bilaterally. The abdomen was soft and non-tender. The results of her hematology report are shown in Table 1. Abnormal values are represented in bold.
A subsequent Chest X-Ray (Figure 1). showed a left density and a preliminary diagnosis of pneumonia was given and antibiotics were started until further imaging was completed.

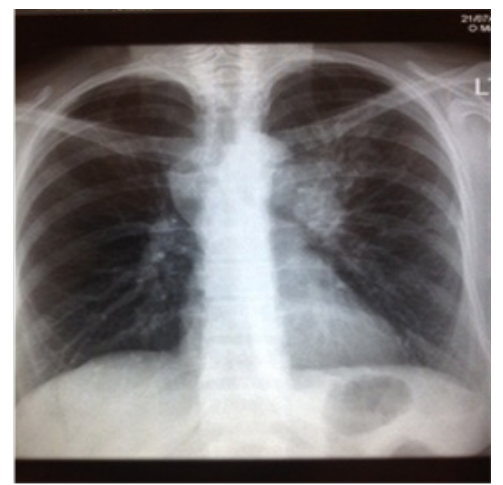

Figure I Chest X Ray illustrating left lung opacity.

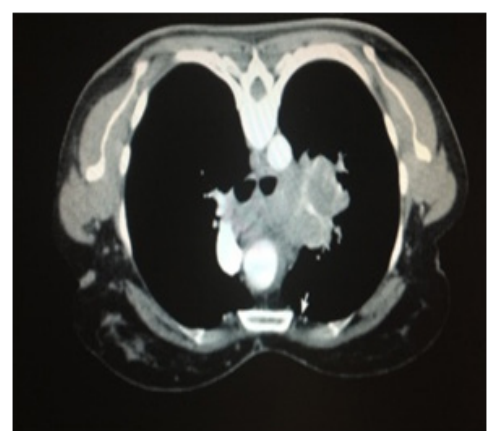

Figure 2 CT Thorax showing a mass encasing left pulmonary artery. 
A subsequent CT Thorax/Abdomen/Pelvis (CT TAP) revealed a large mass encasing the left pulmonary artery with multiple lymph nodes and lesions on the liver (Figure 2). Further ultrasound indicated simple liver cysts (Figure 3). An ensuing Endobronchial ultrasound indicated squamous cell carcinoma with extensive necrosis; this finding was confirmed by bronchial washings. Finally, a PET CT disclosed uptake in the upper lobe with extensive mediastinal involvement, pleural metastasis, and chest wall invasion. There was no liver or bony involvement. The patient was not suitable for surgical treatment and was referred to the Medical Oncology team.

Table I Results of Hematology

\begin{tabular}{cccccccccccccccccc}
\hline WBC & $\mathrm{Hb}$ & $\mathrm{Plt}$ & $\mathrm{ESR}$ & $\mathrm{Na}$ & $\mathrm{K}$ & Urea & $\mathrm{Cr}$ & $\mathrm{Alb}$ & $\mathrm{ALP}$ & Bili & GGT & AST & AST & Ca & CRP & LDH & INR \\
\hline I2.1 & 10.4 & 390 & 90 & 135 & 4 & 1.9 & 32 & 33 & 134 & 9 & 60 & 19 & 28 & 2.32 & 137 & 383 & 1.14 \\
\hline
\end{tabular}

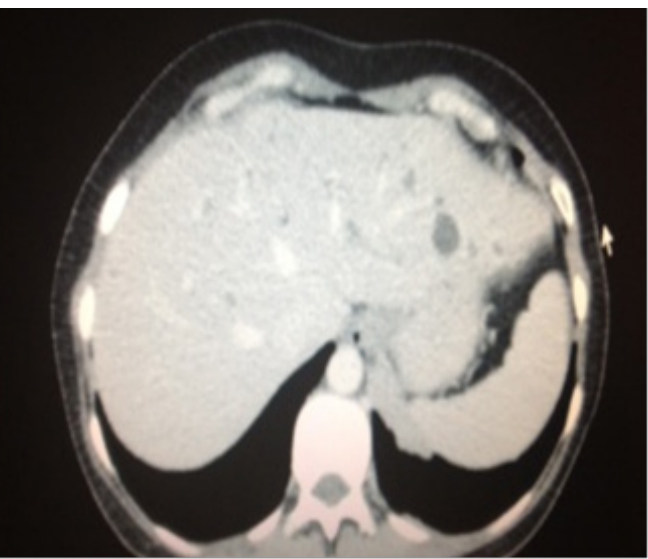

Figure 3: CT Abdomen showing cystic lesions on the liver.

\section{Discussion}

A clinical diagnosis of JPS requires fulfillment of one of the following clinical criteria:two or more histologically confirmed PJ polyps; any number of PJ polyps detected in one individual who has a family history of PJS in close relative(s); mucocutaneous pigmentation in an individual who has a family history of PJS in close relative(s); number of PJ polyps in an individual who also has characteristic mucocutaneous pigmentation. ${ }^{7}$

For patients with a strong positive family history, like our patient, more attention should be given to screening for complications related to the syndrome earlier with or without a current documented PJS diagnosis.

There are a number of current recommendations for PJS cancer screenings. Examples of screening include an initial colonoscopy and upper GI endoscopy at age 8 then repeated every three years in those with significant polyps. For those without significant polyps, surveillance should begin again every three years beginning at age 18. All patients should have screening every $1-2$ years after age $50 .^{7}$ Similar screening for small bowel using video capsule endoscopy is recommended. Women should begin MRI/ultrasound breast screening at age 25 and continue with mammography at $50 .^{7}$

As the link between lung cancers and the STLK1 gene potentially strengthens-more focus could be given to lung screening for patients with PJS. While there is a definite association between this link, not all patients with PJS carry this particular gene. Patients with the LKB1 germline mutation are more likely to develop cancer. ${ }^{2}$ However, patients without the LKB1 mutation and concurrent PJS can still develop lung cancer. ${ }^{8}$ This represents the possibility for an unidentified second disease locus for PJS. With the discovery of more genes responsible for both PJS and its associated cancers better screening and treatment options may become available.

\section{Acknowledgments}

None.

\section{Conflict of interests}

The authors declare no conflict of interests.

\section{References}

1. Parray FQ, Syed AW, Yatoo GN, et al. Peutz-Jeghers Syndrome. North American Journal of Medical Sciences. 2012;4(11):613-614.

2. Forcet C, Etienne Mannevile S, Helene G, et al. Functional analysis of Peutz-Jeghers mutations reveals that the LKB1 C-terminal region exerts a crucial role in regulating both the AMPL pathway and the cell polarity. Human Molecular Genetics. 2005;14(10):1283-1292.

3. Van Lier MGF, Westerman AM, Wagner A, et al. High cancer risk and increased mortality in patients with Peutz-Jeghers syndrome. Gut. 2011;60(2):141-147.

4. Okuda K, Sasaki H, Hikosaka Y, et al. LKB1 Gene alterations in surgically resectable adenocarcinoma of the lung. Surgery Today. 2011;31(1):107-10.

5. Zhong D, Guo L, de Aguirre I, et al. LKB1 Mutation in large cell carcinoma of the lung. Lung Cancer. 2006;53(3)285-94.

6. Herbay A, Arens N, Friedl W, et al. Bronchioloalveolar carcinoma: a new cancer in Peutz-Jeghers syndrome. Lung Cancer. 2005;47(2):283-8.

7. Begg AD, Latchford AR, Vasen HFA, et al. Peutz-Jeghers syndrome: a systematic review and recommendations for management. Gut. 2010;59(7):975-986.

8. Hearle N, Schumacher V, Menko FH, et al. Frequency and spectrum of cancers in the Peutz-Jeghers syndrome. Clinical Cancer Research. 2006;12(10)3209-3215. 